\title{
Approximate mass and radius formulas for static and rotating strange stars
}

\author{
K. S. Cheng* and T. Harko ${ }^{\dagger}$ \\ Department of Physics, University of Hong Kong, Pokfulam Road, Hong Kong, China
}

(Received 27 March 2000; published 26 September 2000)

\begin{abstract}
Using the general relativistic conditions of thermodynamic equilibrium and fitting with values obtained from numerical integration of structure equations we derive approximate mass and radius formulas for static and uniformly rotating strange stars obeying the bag model equation of state. In the static case our formulas can reproduce the values of the mass and radius of the quark star with an error smaller than $1 \%$. The maximum mass and radius of the strange stars can be predicted by fitting only one set of static configuration data. With the use of results obtained for the static configuration and with some supplementary assumptions about the behavior of the metric tensor at the center of the star, we can predict the rotational properties of maximally rotating strange stars within an error of $3 \%$. From the approximate formulas we can derive the "empirical", formula relating the maximum rotation frequency of the uniformly rotating strange stars to the mass and radius of the maximum allowable static configuration.
\end{abstract}

PACS number(s): 97.60.Jd, 12.38.Mh

\section{INTRODUCTION}

It has been argued that strange quark matter, consisting of $u, d$, and $s$ quarks is energetically the most favorable state of quark matter [1,2]. Witten [1] suggested that there are two ways to form strange matter: the quark-hadron phase transition in the early universe and conversion of neutron stars into strange ones at ultrahigh densities. In the theory of strong interactions quark bag models suppose that the breaking of a physical vacuum takes place inside hadrons. As a result vacuum energy densities inside and outside a hadron become essentially different and the vacuum pressure on the bag wall equilibrates the pressure of quarks thus stabilizing the system. If the hypothesis of the quark matter is true, then some of neutrons stars could actually be strange stars, built entirely of strange matter $[3,4]$. However, there are arguments against the existence of strange stars [5].

Several mechanisms have been proposed for the formation of quark stars. For example, strange stars are expected to form during the collapse of the core of a massive star after the supernova explosion [6]. Another possibility is that some rapidly spinning neutron stars in low-mass $\mathrm{x}$-ray binaries (LXMB's) can accrete sufficient mass to undergo a phase transition to become strange stars [7]. Some of the millisecond pulsars may be strange stars because LXMB's are believed to be the progenitors of millisecond pulsars. Strange stars have also been proposed as sources of unusual astrophysical phenomena, e.g., soft $\gamma$-ray repeaters [8], pulsating x-ray burster [9], cosmological $\gamma$-ray bursts $[7,10]$, SAX J1808.4-3658 [11], etc. The mechanism of the phase transition from neutron to quark stars in low LXMB's also results in the excitation of stellar radial oscillations that can be damped by gravitational wave radiation instead of internal viscosity [12]. The discovery of $\mathrm{kHz}$ quasiperiodic oscillation in LXMB's [13] implies that the compact stellar object

\footnotetext{
*Email address: hrspksc@ hkucc.hku.hk

†Email address: tcharko@hkusua.hku.hk
}

must have very soft equation of state, which is consistent with that of strange stars [14].

Assuming that interactions of quarks and gluons are sufficiently small the energy density $\epsilon=\rho c^{2}$ and pressure $p$ of a quark-gluon plasma at temperature $T$ and chemical potential $\mu_{f}$ (the subscript $f$ denotes the various quark flavors $u, d, s$, etc.) can be calculated by thermal theory. Neglecting quark masses and supposing that quarks are confined to the bag volume (in the case of a bare strange star, the boundary of the bag coincides with stellar surface), the equation of state is

$$
p=\frac{(\epsilon-4 B)}{3}
$$

where $B$ is the difference between the energy density of the perturbative and nonperturbative QCD vacuum (the bag constant). Equation (1) is essentially the equation of state of a gas of massive particles with corrections due to the QCD trace anomaly and perturbative interactions. These are always negative, reducing the energy density at given temperature by about a factor of 2 [15]. This equation of state does not depend upon quark flavor number, hence it will be correct either for strange quark matter $\left(m_{s} \rightarrow 0\right)$ or for normal quark matter $\left(m_{s} \rightarrow \infty\right)$. For any intermediate values of $m_{s}$ the equation of state gives the pressure with error less than $4 \%$ [5]. Thus the equation of state of strange matter is mainly determined by the vacuum energy density $B$.

The equation of state (1) has been the basis for the study of most of the static relativistic models of strange stars. A complete description of static strange stars has been obtained based on numerical integration of mass continuity and Tolman-Oppenheimer-Volkov (TOV) (hydrostatic equilibrium) equations for different values of the bag constant $[3,4]$. Using numerical methods the maximum gravitational mass $M_{\text {max }}$, the maximum baryon mass $M_{B, \max } \equiv 1.66 \times 10^{-27} \mathrm{~kg}$ $\times N_{B}\left(N_{B}\right.$ is the total baryon number of the stellar configuration), and the maximum radius $R_{\max }$ of the strange star, have been obtained, as a function of the bag constant, in the form $[1,4,16-18]$ 


$$
\begin{aligned}
& M_{\max }=\frac{1.9638 M_{\odot}}{\sqrt{B_{60}}}, \quad M_{B \max }=\frac{2.6252 M_{\odot}}{\sqrt{B_{60}}}, \\
& R_{\max }=\frac{10.172 \mathrm{~km}}{\sqrt{B_{60}}},
\end{aligned}
$$

where $B_{60} \equiv B /\left(60 \mathrm{MeV} \mathrm{fm}^{-3}\right)$.

In the slow rotation approximation the rotational properties of strange stars have been investigated in [19] and [20]. As far as rotational deformations are concerned, there are a number of detailed differences between the strange star models and standard neutron stars. Exact numerical calculations of rapidly rotating strange stars were done by $[18,21,22]$. Rotation increases the maximum allowable mass of strange stars and the equatorial radius of the maximum mass configuration. Reference [18] obtained for the maximum mass and radius of quark stars the following two formulas:

$$
M_{\max }^{\mathrm{rot}}=\frac{2.831 M_{\odot}}{\sqrt{B_{60}}}, \quad R_{\mathrm{eq}, M_{\max }}^{\mathrm{rot}}=\frac{16.54 \mathrm{~km}}{\sqrt{B_{60}}} .
$$

Because of the nonspherical symmetry of rapidly rotating stationary stellar configurations and in view of the complicated character of the interplay of the effects of rotation and of those of general relativity, at first sight it seems difficult to find a simple universal relation between the mass and radius of nonrotating configurations with maximum allowable mass $M_{\max }^{\text {stat }}$ and radius $R_{\max }^{\text {stat }}$ and the maximum rotation frequency. However, $[16,17]$ have found a simple relation connecting the maximum rotation frequency $\Omega_{\max }$ with the maximum mass and radius of the static configuration

$$
\Omega_{\max }=C\left(\frac{M_{\max }^{\text {stat }}}{M_{\odot}}\right)^{1 / 2}\left(\frac{R_{\max }^{\mathrm{stat}}}{10 \mathrm{~km}}\right)^{-3 / 2},
$$

with $C$ a constant which does not sensitively depend on the equation of state of dense matter. Equation (4) has been checked by [23] for 13 equations of state of dense matter and by [24] for 12 realistic equations of state. The value of the constant $C$ has been obtained by fitting equation (4) with data obtained by numerically integrating the gravitational field equations. It is given by $C=7700 \mathrm{~s}^{-1}$ [23] or by $C$ $=7730 \mathrm{~s}^{-1}$ [18]. In the case of strange stars the empirical formula holds with a very good precision, the relative deviation not exceeding $2 \%$ [18].

It is the purpose of the present paper to derive approximate mass and radius formulas for general relativistic static and rotating equilibrium strange matter configurations described by the bag model equation of state (1). In order to do this only the general relativistic thermodynamic equilibrium conditions are used. In the case of the static star the two arbitrary integration functions involved are obtained by fitting the obtained representations for mass and radius with the data resulted from the numerical integration of the TOV and mass continuity equations. The final relations give the mass and radius of a strange star as a function of the central density and of the bag constant. They exactly describe the basic physical parameters (mass and radius) of strange stars from the case of strange matter at zero pressure, $\rho_{c}=4 B$, up to the upper limit of stability of the quark star, $M=2 M_{\odot}$. The results obtained for static configurations are extended to rotating strange stars whose surface is described by the Kerr line element. Imposing the condition of the thermodynamic equilibrium mass-radius formulas are obtained that depend on three unknown functions. An appropriate (but empirical) choice of these parameters leads to the description of the mass and radius of rapidly rotating equilibrium strange star configurations within a mean precision of $3 \%$. As an application of the obtained analytical representations for mass and radius we give a derivation of the "empirical" relation (4) and we calculate the expression of the total energy of the static strange star. Obtaining general relations for mass and radius of stars is also of practical importance. For example, the existence of such relations enables rapid and precise calculations of the upper bounds of global parameters of stellar structures.

The paper is organized as follows. In Sec. II, the basic formalism is described and mass and radius formulas for static quark stars are obtained. In Sec. III we discuss rotating strange matter configurations with the vacuum boundary described by the Kerr geometry. In Sec. IV we discuss and conclude our results.

\section{APPROXIMATE MASS AND RADIUS FORMULAS FOR STATIC STRANGE STARS}

The changes caused by the general theory of relativity in the conditions of thermal equilibrium are of fundamental importance. In a constant gravitational field we must distinguish the conserved energy $E_{0}$ of any small part of the stellar object from the energy $E$ measured by an observer situated at a given point. These two quantities are related by $E_{0}$ $=E \sqrt{g_{00}}$ [25], where $g_{00}$ is the time component of the metric tensor. A similar change occurs in the condition of the constancy of the chemical potential $\mu=(\partial E / \partial N)_{S, V}$ throughout the star. Since the particle number $N$ is a constant for the stellar object, at any point inside the gravitating body the chemical potential obeys the relation $\mu \sqrt{g_{00}}=$ const [26].

Since we suppose that the strange star is in thermal equilibrium a similar relation also holds for the temperature $T$, $T \sqrt{g_{00}}=$ const [26]. Consequently, $\mu / T=$ const inside the compact object and $d \mu / \mu=d T / T$. At constant volume (equal to unity) we have $d p=s d T+n d \mu$, where $s$ and $n$ are the entropy and number of particles in unit volume of the body, respectively. With the use of $d T=T(d \mu / \mu)$ and taking into account that $\mu n+s T=\epsilon+p$ we obtain the following equation relating the chemical potential to the energy density and pressure of the star [26]:

$$
\frac{d \mu}{\mu}=\frac{d p}{\epsilon+p} .
$$

Consider now a static equilibrium quark matter configuration satisfying the bag model equation of state (1). Let us compare the values of the chemical potential $\mu=\Sigma \mu_{f}$ at two points: at the center of the star and at the vacuum boundary. From $\mu \sqrt{g_{00}}=$ const we obtain 


$$
\mu_{C}\left(\sqrt{g_{00}}\right)_{C}=\mu_{S}\left(\sqrt{g_{00}}\right)_{S}
$$

where the indices $C$ and $S$ refer to the center and to surface of the quark star, respectively. At the vacuum boundary the gravitational field of the strange star is described by the Schwarzschild solution, which gives $g_{00}=1_{S}-2 G M / c^{2} R$ [25], where $M$ and $R$ are the total mass and radius of the strange star, respectively. In the following we denote the time component $g_{00}$ of the metric tensor at the center of the strange star by $g_{00} l_{C}=C\left(\rho_{C}, B\right)$.

From a physical point of view $C\left(\rho_{C}, B\right)$ can be related via the relation $1 / C-1=z_{c}$ to the redshift $z_{c}$ of a photon emitted from the center of the quark star. For a given static strange matter configuration the value of the function $C$ depends only on the central density of the quark star $\rho_{C}$ and on the bag constant $B$. Therefore, from Eq. (6) we obtain

$$
\frac{\mu_{C}^{2}}{\mu_{S}^{2}}=\frac{1}{C\left(\rho_{C}, B\right)}\left(1-\frac{2 G M}{c^{2} R}\right) .
$$

With the use of the bag model equation of state (1) we can integrate Eq. (5) to obtain $\mu=C_{0}(\epsilon-B)^{1 / 4}$. The integration constant $C_{0}$ can be determined by calculating the chemical potential at the center of the quark star. Hence we obtain $C_{0}=\mu_{C} /\left(\epsilon_{C}-B\right)^{1 / 4}$ and the variation of the chemical potential inside the quark star can be represented as

$$
\mu=\frac{\mu_{C}}{\left(\epsilon_{C}-B\right)^{1 / 4}}(\epsilon-B)^{1 / 4} .
$$

At the surface of the star $\epsilon_{S} \rightarrow 4 B$. Therefore, from Eq. (8) it follows that

$$
\mu_{S}=(3 B)^{1 / 4} \frac{\mu_{C}}{\left(\epsilon_{C}-B\right)^{1 / 4}} .
$$

In order to simplify the notation we introduce a dimensionless parameter $\eta=\rho_{C} / B$, so that $C\left(\rho_{C}, B\right)=C(\eta)$. Eliminating $\mu_{C} / \mu_{S}$ from Eqs. (7) and (9) we obtain the following exact formula for the mass-radius ratio of a strange star:

$$
\frac{M}{R}=\frac{c^{2}}{2 G}\left[1-C(\eta)\left(\frac{\eta-1}{3}\right)^{1 / 2}\right]
$$

For a given equation of state the mass-radius ratio of the star depends on the values of the metric tensor component at the center of the star $C(\eta)$, only. A possible representation for the function giving the values of $g_{00}$ at the center of the quark star is in the form of a power series $C(\eta)=$ const $+\Sigma_{i}\left(a_{i} / \eta^{i}\right)$, with $a_{i}$ constants.

As applied on the star surface the mass continuity equation leads to a rough approximation of the quark star mass of the form $d M / d r \approx M / R \sim 16 \pi B R^{2}$. A mass-radius relation of this form could also describe zero-pressure quark matter, with $p=0$ and $\rho=\rho_{C}=4 B$. But for densities greater than $4 B$ the effects determined by the large central density become important. Hence for strange quark stars we propose the following mass-radius relation:

$$
M=\frac{16 \pi B}{3} a(\eta) R^{3}
$$

with $a(\eta)$ a function describing the variation in the quark star mass due to the increase of the central density.

The exact form and the values of the functions $C(\eta)$ and $a(\eta)$ can be determined only by numerically integrating the gravitational field equations. By fitting the numerical data [27] for the mass and radius of the strange star with the expressions (10) and (11) we obtain the following representations for these functions (in the present paper we consider $\left.B=10^{14} \mathrm{~g} / \mathrm{cm}^{3}=56 \mathrm{MeV} / \mathrm{fm}^{3}\right)$ :

$$
\begin{aligned}
C(\eta)= & 44.005 \frac{1}{\eta^{3}}-6.68158 \frac{1}{\eta^{2}}+2.7403 \frac{1}{\eta}+0.0554667 \\
a(\eta)= & 0.0000521833 \eta^{3}-0.00378523 \eta^{2} \\
& +0.11456 \eta+0.624094
\end{aligned}
$$

The numerical constants in Eqs. (12) and (13) depend on $B$ because the numerical data have been calculated at a given $B$. For the polynomial fittings (12) and (13) the correlation coefficient $r=0.9997$ and the probability $P<0.001$. Therefore, for a given value of the bag constant $B$ we obtain the following exact representations for the radius and mass of the static strange matter configuration obeying the MIT bag model equation of state:

$$
\begin{aligned}
R_{\text {stat }}(\eta)= & \left(\frac{3 c^{2}}{32 \pi G B}\right)^{1 / 2}\left[\frac{1}{a(\eta)}\left(1-C(\eta)\left(\frac{\eta-1}{3}\right)^{1 / 2}\right)\right]^{1 / 2}, \\
M_{\text {stat }}(\eta)= & \frac{\sqrt{3}}{2}\left(\frac{c^{2}}{32 \pi G B}\right)^{1 / 2}(a(\eta))^{-1 / 2} \\
& \times\left[1-C(\eta)\left(\frac{\eta-1}{3}\right)^{1 / 2}\right]^{3 / 2} .
\end{aligned}
$$

Here, we want to emphasize that Eqs. (14) and (15) become incorrect as $\rho_{C} \rightarrow 4 B$, which corresponds to the zero-pressure situation.

The variations of the radius and mass for a strange star as a function of the parameter $\eta\left(B=10^{14} \mathrm{~g} / \mathrm{cm}^{3}\right)$ are represented in Figs. 1 and 2. For the sake of comparison we have also presented the data obtained by numerically integrating the Tolman-Oppenheimer-Volkov (TOV) and hydrostatic equilibrium equations [27]. Using Eqs. (12)-(15) we can reproduce the values of the mass and radius of the quark star obtained by numerical integration with an error smaller than $1 \%$. The maximum radius of the strange star is obtained from the condition $d R / d \eta=0$. The corresponding algebraic equation has the solution $\eta_{\max }^{R}=9.99012$ (this value depends of course on the value of $B$ ), giving the value of the ratio of the central pressure and bag constant for the maximum allowable radius $R_{\max }$ of the static strange star. This can be expressed as 


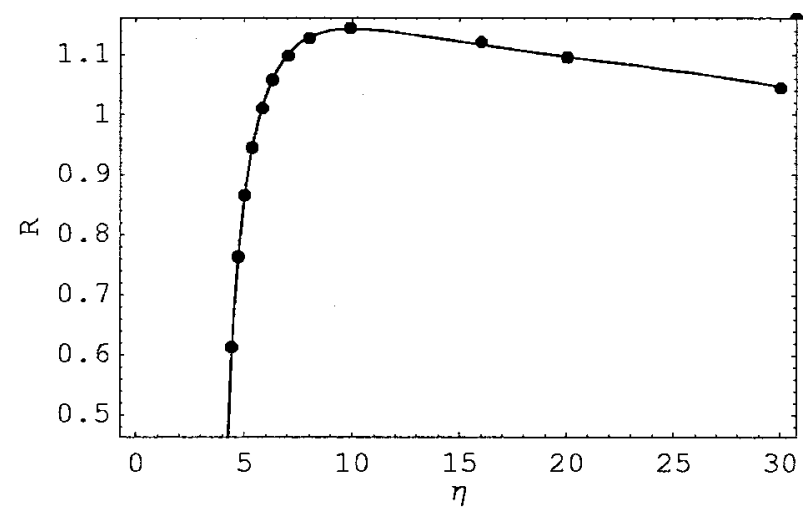

FIG. 1. Variation of the radius $R$ (in units of $10^{6} \mathrm{~cm}$ ) of the static strange star as a function of the parameter $\eta=\rho_{C} / B$. The solid curve represents the values of the radius calculated with Eq. (14), while the points represent the values of the radius of the quark star obtained by numerically integrating the general relativistic structure equations.

$$
R_{\max }^{\mathrm{stat}}=0.569906 \times \sqrt{\frac{3 c^{2}}{32 \pi B G}},
$$

and its numerical value for $B=10^{14} \mathrm{~g} / \mathrm{cm}^{3}$ is $R_{\max }=1.1436$ $\times 10^{6} \mathrm{~cm}$. From the condition $d M / d \eta=0$ it follows that $\eta_{\max }^{(M)}=22.41173$ and the maximum mass of the static quark star is given by

$$
M_{\max }^{\mathrm{stat}}=0.297866 \times \frac{\sqrt{3}}{2(32 \pi B)^{1 / 2}}\left(\frac{c^{2}}{G}\right)^{3 / 2} .
$$

From Eq. (17) and for the chosen value of the bag constant, we obtain a value of $M_{\max }=2.016 M_{\odot}$. These results are in good agreement with the previously proposed $[1,4]$ maximum radius and mass values, given by Eq. (2) [from Eq. (2) and for $B=56 \mathrm{MeV} / \mathrm{fm}^{3}$ we obtain $\left.M_{\max }=2.03 M_{\odot}\right]$.

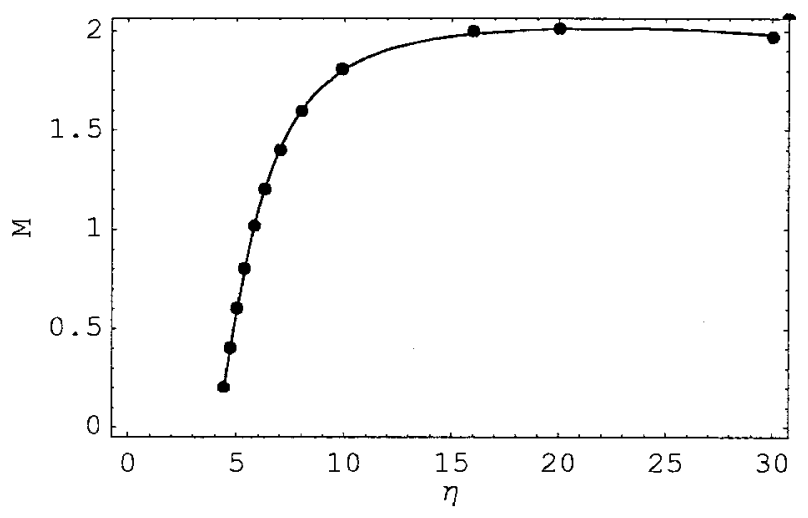

FIG. 2. Variation of the mass $M$ (in solar mass units) of the static strange star as a function of the parameter $\eta$. The solid curve represents the values of the mass calculated with Eq. (15), while the points represent the values of the mass of the strange star obtained by numerically integrating the general relativistic structure equations.
For values of $\eta>\eta_{\max }^{(M)}$ static quark star models would be unstable to radial perturbations.

\section{ROTATING EQUILIBRIUM STRANGE STAR CONFIGURATIONS}

Rotation is a basic property of stellar objects. The general relativistic theory of rapidly rotating objects is of great intrinsic interest because of its important applications in astrophysics. An analytic vacuum solution to the Einstein gravitational field equations, the Kerr metric [28], which is asymptotically flat and has the general properties expected of an exterior metric of a rotating object has been known for many years. But up to now attempts to fit a perfect fluid interior solution to this exterior metric have been unsuccessful. The study of the rotation of neutron or strange stars has been done mainly using refined numerical methods. Since it is expected that the quark star should be associated with LXMB's (one of which has been confirmed to contain a millisecond compact object $[29,30])$ the study of the rotational properties of strange stars can be used to constrain the equation of state of the ultradense matter.

To describe the interior of the rotating quark star we adopt the formalism of $[18,31]$. Under the hypothesis of stationary, axial symmetry and purely azimuthal motion a coordinate system $(t, r, \theta, \phi)$ can be chosen so that inside the star the line element takes the form

$$
\begin{aligned}
d s^{2}= & N^{2} c^{2} d t^{2}-B^{2} r^{2} \sin ^{2} \theta\left(d \phi-N^{\phi} c d t\right)^{2} \\
& -A^{2} d r^{2}+r^{2} d \theta^{2},
\end{aligned}
$$

where $N, N^{\phi}, A$, and $B$ are functions of $r$ and $\theta$ only. The fluid 3 velocity, as measured by the locally nonrotating observer, is given by $U=B r \sin \theta\left[\left(\Omega-N^{\phi}\right) / N\right]$, where $\Omega$ $=d \phi / d t$ is the angular velocity of a fluid element moving in the $\phi$ direction (physically it is the angular velocity as measured by an observer at spatial infinity) [18,31]. From the point of view of our thermodynamic approach the most important result is the equation of the stationary motion, which results from the Bianchi identities and which for a perfect fluid reduces to [31]

$$
\frac{1}{\epsilon+p} \frac{\partial p}{\partial x^{i}}+\frac{\partial \nu}{\partial x^{i}}-\frac{\partial}{\partial x^{i}}(\ln \Gamma)=-F \frac{\partial \Omega}{\partial x^{i}}
$$

where we denoted $F=\Gamma^{2}\left(A^{2} B / N\right) U r \sin \theta, \nu=\ln N$, and $\Gamma$ $=\left(1-U^{2} / c^{2}\right)^{-1 / 2}$. If $\Omega=$ const (case called uniform or rigid rotation) and with the use of the bag equation of state (1), Eq. (19) can be integrated to give the following fundamental result describing the stationary equilibrium of a rotating strange star:

$$
\begin{aligned}
{[2 \nu} & \left.+\frac{1}{2} \ln (\epsilon-B)-\ln \left(1-\frac{U^{2}}{c^{2}}\right)\right]_{\text {center }} \\
& =\left[2 \nu+\frac{1}{2} \ln (\epsilon-B)-\ln \left(1-\frac{U^{2}}{c^{2}}\right)\right]_{\text {surface }} .
\end{aligned}
$$


Equation (20) is the generalization to the case of rotation of the well-known static Bianchi identity $d \nu / d r=-2 /(\epsilon$ $+p) d p / d r[25]$.

The metric outside rotating stars has been computed numerically for both neutron and quark star configur- ations. But for the sake of simplicity in the present paper we assume that the vacuum boundary of the rotating strange star is described by the Kerr metric [28] in the Boyer-Lindquist coordinates, which has the form $[32,33]$ :

$$
\begin{aligned}
d s^{2}= & \left(1-\frac{2 G M r / c^{2}}{r^{2}+a^{2} \cos ^{2} \theta / c^{2}}\right) c^{2} d t^{2}-\frac{r^{2}+a^{2} \cos ^{2} \theta / c^{2}}{r^{2}+a^{2} / c^{2}-2 G M r / c^{2}} d r^{2}-\left(r^{2}+a^{2} \cos ^{2} \theta / c^{2}\right) d r^{2}-\left(r^{2}+a^{2} \sin ^{2} \theta / c^{2}\right) d \theta^{2} \\
& -\left[\left(r^{2}+a^{2} / c^{2}\right) \sin ^{2} \theta+\frac{2 G M r a^{2} \sin ^{4} \theta / c^{2}}{r^{2}+a^{2} \cos ^{2} \theta / c^{2}}\right] d \phi^{2}-2 \frac{2 G M r a \sin ^{2} \theta / c^{2}}{r^{2}+a^{2} \cos ^{2} \theta / c^{2}} c d t d \phi .
\end{aligned}
$$

In this form the Kerr metric is manifestly axially symmetric and closely resembles the Schwarzschild solution in its standard form. $M$ is the mass of the source and the parameter $a$ is the ratio between the angular momentum $J$ and the mass of the rotating quark matter configuration: $a=-J / M$. The negative sign in the definition of $a$ should be stressed: a body rotating in a positive sense will have a positive $J$ and a negative $a$ [32].

We apply Eq. (20) at two points: at the center of the dense core and at the pole of the rotating quark star. At the center of the star and along the rotation axis $U=0$. We denote $C_{\text {rot }}^{(1)}$ as the value of the metric tensor component $g_{00}$ at the center of the star. At the polar point $\theta=0$ and $r=R_{p}=$ const, where $R_{p}$ is the polar radius of the star. Therefore, in this point the line element is given by

$$
d s_{\mathrm{pol}}^{2}=\left(1-\frac{2 G M}{c^{2} R_{p}} \frac{1}{1+\frac{a^{2}}{c^{2} R_{P}^{2}}}\right) c^{2} d t^{2} .
$$

From the definition of the angular momentum $J=I \Omega$, where $I$ is the moment of inertia of the star. Consequently, from Eq. (20) we obtain the following exact mass-polar radius relation for strange stars:

$$
\frac{M}{R_{p}}=\frac{c^{2}}{2 G}\left(1+\frac{a^{2}}{c^{2} R_{p}}\right)\left(1-C_{\mathrm{rot}}^{(1)} \sqrt{\frac{\eta-1}{3}}\right) .
$$

Let us apply now Eq. (20) for two points situated in the equatorial plan of the rotating quark star: at the center of the star and at the equator, respectively. At the equator $r=R_{e}$ $=$ const and $\theta=\pi / 2\left(R_{e}\right.$ is the equatorial radius of the star). Rotation in general changes the distribution of the mass in the body and the moment of inertia is a function of the angular velocity $\Omega$. But as a first approximation we shall define it via the Newtonian expression $I=\frac{2}{5} M R_{e}^{2}$, then $a$ $=-\frac{2}{5} R_{e}^{2} \Omega$. For a uniform rotation the rotation angle of the source and/or observer at the equator is $\phi=\Omega t$. Taking into account these results we obtain the Kerr metric at the equator of the rotating strange star in the form

$$
d s_{\mathrm{eq}}^{2}=\left[1-\frac{2 G M}{c^{2} R_{e}}\left(1-\frac{a \Omega}{c^{2}}\right)^{2}-\frac{R_{e}^{2} \Omega^{2}}{c^{2}}\left(1+\frac{a^{2}}{c^{2} R_{e}^{2}}\right)\right] c^{2} d t^{2} .
$$

With the use of Eqs. (20) and (23) we obtain the following exact mass-equatorial radius relation for rotating strange stars:

$$
\frac{M}{R_{e}}=\frac{c^{2}}{2 G}\left[\frac{1-C_{\mathrm{rot}}^{(2)}\left(\eta, \Omega, R_{e}\right) \sqrt{\frac{\eta-1}{3}}-\frac{R_{e}^{2} \Omega^{2}}{c^{2}}\left(1+\frac{4}{25} \frac{R_{e}^{2} \Omega^{2}}{c^{2}}\right)}{\left(1-\frac{2}{5} \frac{R_{e}^{2} \Omega^{2}}{c^{2}}\right)^{2}}\right] .
$$

In Eq. (24) we have defined a new arbitrary function $C_{\mathrm{rot}}^{(2)}\left(\eta, \Omega, R_{e}\right)=C_{\mathrm{rot}}^{(1)}\left(\eta, \Omega, R_{e}\right)\left[\left(1-U^{2} / c^{2}\right)^{-1 / 2}\right]_{r=R_{e}} . \quad \mathrm{We}$ denote the ratio of the polar radius and equatorial radius (the oblateness of the star) by $e, e=R_{p} / R_{e}$. For a rapidly rotating strange star we assume that the condition $a^{2} / c^{2} R_{p}^{2}=(4 / 25) e^{2} R_{e}^{2} \Omega^{2} \ll 1$ holds. Therefore, from Eqs. (22) and (24) we obtain the oblateness of the star in the form 


$$
\begin{aligned}
e & =\frac{R_{p}}{R_{e}} \\
& =\frac{1-C_{\mathrm{rot}}^{(2)}\left(\eta, \Omega, R_{e}\right) \sqrt{\frac{\eta-1}{3}}-\frac{R_{e}^{2} \Omega^{2}}{c^{2}}\left(1+\frac{4}{25} \frac{R_{e}^{2} \Omega^{2}}{c^{2}}\right)}{\left(1-\frac{2}{5} \frac{R_{e}^{2} \Omega^{2}}{c^{2}}\right)^{2}\left(1-C_{\mathrm{rot}}^{(1)}\left(\eta, \Omega, R_{e}\right) \sqrt{\frac{\eta-1}{3}}\right)} .
\end{aligned}
$$

In the case of the static strange star we have proposed Eq. (11) as the mass-radius relation. We generalize this relation to the rotating case by assuming that the mass of the rotating strange star is related to its equatorial radius and to the parameter $e$ by

$$
M=\frac{16 \pi B}{3} R_{e}^{3} C_{\mathrm{rot}}^{(3)}\left(\eta, \Omega, R_{e}\right) e,
$$

with $C_{\mathrm{rot}}^{(3)}\left(\eta, \Omega, R_{e}\right)$ being a function describing the combined general relativistic effects due to rotation and variation of central density. In the static case $R_{p}=R_{e}, e=1$, and Eq. (26) reduces to Eq. (11). Equations (22) and (24) - (26) give a complete description of the mass and radius of the rotating strange star.

The present approach, which is basically thermodynamic in its essence, cannot predict the exact form and the values of the functions $C_{\text {rot }}^{(i)}, i=1,2,3$. The only thing we can do is to assume, also based on the static case, some empirical forms for these functions and to check if the resulting formulas can give a satisfactory description of rotating strange star configurations. Therefore, we propose the following approximate empirical expressions for these unknown parameters:

$$
\begin{aligned}
& C_{\mathrm{rot}}^{(1)}\left(\eta, \Omega, R_{e}\right) \approx \frac{1}{2}\left[9\left(1-\frac{R_{e}^{2} \Omega^{2}}{c^{2}}\right)-7\right] C(\eta), \\
& C_{\mathrm{rot}}^{(2)}\left(\eta, \Omega, R_{e}\right)=\left(1-\frac{R_{e}^{2} \Omega^{2}}{c^{2}}\right)^{3 / 2} C(\eta), \\
& C_{\mathrm{rot}}^{(3)}\left(\eta, \Omega, R_{e}\right)=a(\eta),
\end{aligned}
$$

the best fitting with the numerical data is obtained for

$$
C_{\mathrm{rot}}^{(1)}\left(\eta, \Omega, R_{e}\right)=\left[4.46346\left(1-\frac{R_{e}^{2} \Omega^{2}}{c^{2}}\right)-3.432\right] C(\eta) .
$$

Here $C(\eta)$ and $a(\eta)$ are the functions corresponding to the static case given by Eqs. (12) and (13). We compare our results obtained with the use of Eqs. (22) and (24)-(29) with the results provided by [22] obtained by numerically integrating the gravitational field equations for maximally rotating strange stars. The results of [22] are also in very good agreement with the results of the exact numerical models of rotating strange stars built of self-bounded quark matter of [18], the difference between these two works being smaller than $1 \%$. In Fig. 3 we have represented the variation

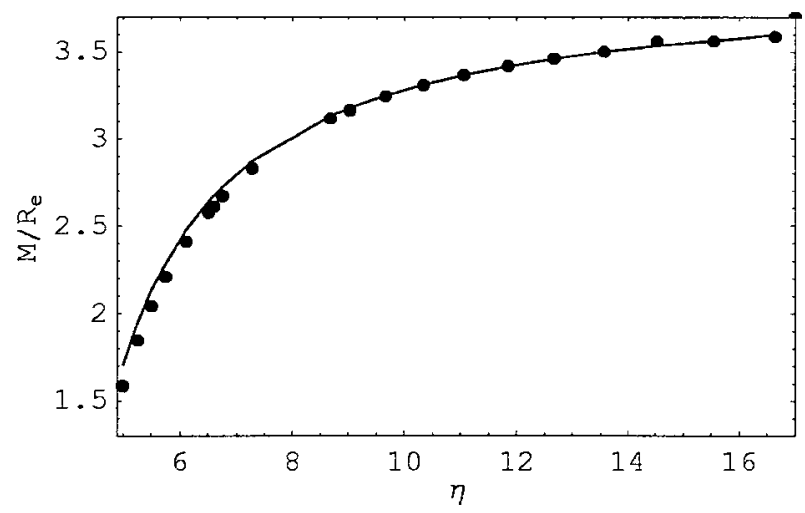

FIG. 3. Mass-equatorial radius ratio (in units of $10^{27} \mathrm{~g} / \mathrm{cm}$ ) of rotating strange stars as a function of the central density of the star, for values of the angular velocity in the range 7049-10030 s${ }^{-1}$. Values obtained from the analytical expression (solid curve) are compared with data obtained by Stergioulas et al. [22] numerically integrating the gravitational field equations (points).

of the mass-equatorial ratio for rotating strange stars, given by Eq. (24) and the values presented in [22], calculated for the same values of the central density and angular velocity. The mean of the difference between these two sets of values is smaller than $2 \%$. In Fig. 4 we present the oblateness parameter $e$. The values obtained with the use of Eq. (25) also agree with the ones obtained by numerically integrating the gravitational field equations within a mean error range of $2 \%$. Figure 5 represents the variation of the mass of the rapidly rotating strange matter configuration in the two approaches.

From Eqs. (24) and (26) we obtain the following representation of the equatorial radius of the rotating strange star as a function of the central density and angular velocity only:

$$
R_{e} \approx c \sqrt{\frac{6(1-C(\eta) \sqrt{(\eta-1) / 3})}{64 \pi G B a(\eta)-27 \Omega^{2} C(\eta) \sqrt{(\eta-1) / 3}}} .
$$

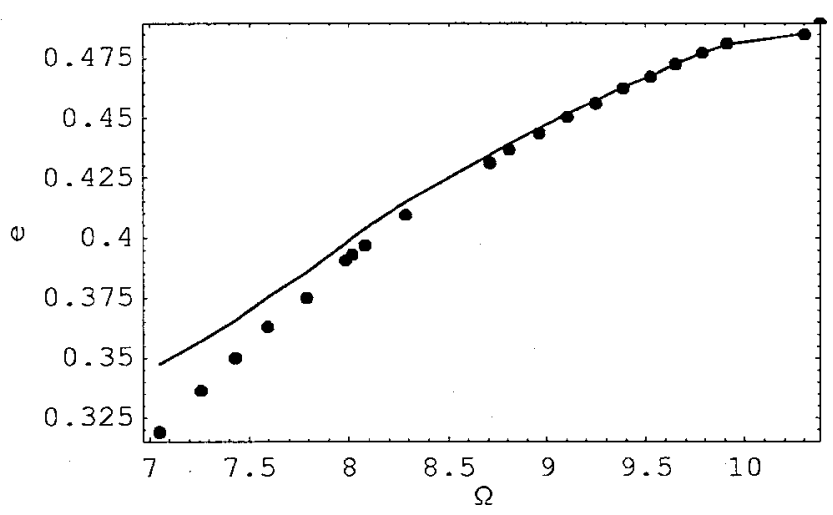

FIG. 4. Polar radius-equatorial radius ratio for rotating strange stars as a function of the angular velocity (in units of $10^{3} \mathrm{~s}^{-1}$ ), for values of the central density in the range $\eta \in[5,16.63]$. Values obtained from the analytical expression (solid curve) are compared with data obtained by Stergioulas et al. [22] numerically integrating the gravitational field equations (points). 


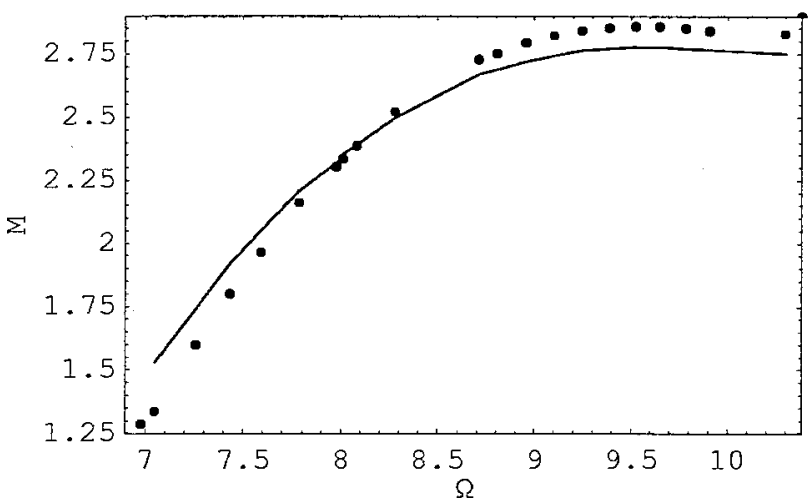

FIG. 5. The mass of maximally rotating strange stars expressed in solar mass units, as a function of the angular velocity (in units of $\left.10^{3} \mathrm{~s}^{-1}\right)$, for values of the central density in the range $\eta$ $\in[5,16.63]$. Values obtained from the analytical expression (solid curve) are compared with data obtained by Stergioulas et al. [22] numerically integrating the gravitational field equations (points).

The equatorial radius can be expressed as a function of mass and radius of the static configuration in the form

$$
R_{e} \approx\left(\frac{M_{\text {stat }}}{R_{\text {stat }}}\right)^{1 / 2}\left[\frac{M_{\text {stat }}}{R_{\text {stat }}^{3}}-\frac{9 \Omega^{2}}{4 G}\left(1-\frac{2 G M_{\text {stat }}}{c^{2} R_{\text {stat }}}\right)\right]^{-1 / 2} .
$$

The equatorial radius is defined only for values of the angular velocity satisfying the condition

$$
\frac{9 \Omega^{2}}{4 G}\left(1-\frac{2 G M_{\text {stat }}}{c^{2} R_{\text {stat }}}\right) \leqslant \frac{M_{\text {stat }}}{R_{\text {stat }}^{3}} .
$$

Therefore, we obtain for the maximum admissible angular velocity of the uniformly rotating strange star the relation

$$
\Omega_{\max }=C_{Q S}\left(1-\frac{2 G M_{\mathrm{stat}}^{\max }}{c^{2} R_{\mathrm{stat}}^{\max }}\right)^{-1 / 2}\left(\frac{M_{\mathrm{stat}}^{\max }}{M_{\odot}}\right)^{1 / 2}\left(\frac{R_{\max }^{\mathrm{stat}}}{10 \mathrm{~km}}\right)^{-3 / 2} \text {, }
$$

where $C_{Q S}=2 / 3 \sqrt{G}\left[\left(M_{\odot}\right)^{1 / 2} / 10^{9}\right]=7699 \mathrm{~s}^{-1}$.

Equation (32) is very similar to the "empirical" formula, given by Eq. (4), obtained by $[16,17]$ and further discussed by $[23,24]$. The coefficient of proportionality in Eq. (32) is independent of the equation of state of the dense matter. The concordance of the numerical value of the constant $C_{Q S}$ obtained in this derivation and the values of $[18,23]$ is very good, the difference being smaller than $1 \%$. Previous attempts to derive the "empirical" relation (4) in the slow rotation limit, but without satisfactory results, are those of [20] and [34,35].

Substituting the radius given by Eq. (30) into Eq. (26) we obtain an approximate mass equation relating the mass of rotating strange star to its central density and to the angular velocity only. But this equation has a very complicated algebraic form and we shall not present it here. The investigation of the maximum mass and radius of the rotating strange star can be done even by using the exact formulas obtained above only by numerical methods and leads to results very similar to those published in the previous studies.

The previous results can be also extended to study stellar objects having strong gravitational fields but uniform and slow rotation (slow as compared to the critical angular velocity $\Omega_{c}=\left(G M / R^{3}\right)^{1 / 2}$ of the centrifugal breakup). Slow rotation has usually been considered by using the prescription of [36]. Homogeneous neutron stars rotating at the secular instability limit $\Omega=0.52 \Omega_{c}$, which is relevant for pulsars, are within this bound [37]. For a slowly rotating body the exterior line element can be obtained by neglecting the terms proportional to $1 / r^{2}$ in the Kerr metric [38]. Using the condition of thermodynamic equilibrium and fitting to the results of numerical investigations or using some supplementary assumptions, high precision mass and radius formulas for slowly rotating strange stars can also be constructed.

\section{DISCUSSIONS AND FINAL REMARKS}

As an application of the mass and radius formulas obtained for the static strange stars we shall derive an explicit expression for the total energy of the quark star. The total energy (including the gravitational field contribution) inside an equipotential surface $S$ can be defined, according to $[39,40]$ to be

$$
E=E_{M}+E_{F}=\frac{c^{4}}{8 \pi G} \xi_{s} \int_{S}[K] d s
$$

where $\xi^{i}$ is a Killing vector field of time translation, $\xi_{s}$ is its value at $S$, and $[K]$ is the jump across the shell of the trace of the extrinsic curvature of $S$, considered as embedded in the 2-space $t=$ const. $E_{M}=\int_{S} T_{i}^{k} \xi^{i} \sqrt{-g} d S_{k}$ and $E_{F}$ are the energy of the matter and of the gravitational field, respectively. This definition is manifestly coordinate invariant. For a static strange star we obtain for the total energy (also including the gravitational contribution) the following expression:

$$
\begin{aligned}
E=E_{S Q M}+E_{F}= & -\frac{c^{5}}{(G)^{3 / 2}} \sqrt{\frac{3}{32 \pi B}} C(\eta) \\
& \times\left[\frac{1}{a(\eta)} \frac{\eta-1}{3}\left(1-C(\eta) \sqrt{\frac{\eta-1}{3}}\right)\right]^{1 / 2},
\end{aligned}
$$

where $E_{S Q M}$ is the total energy of the quark matter.

The variation of the total energy of the strange star as a function of the parameter $\eta$ is represented in Fig. 6. The minimum value of the total-matter plus gravitational-energy of the strange matter configuration is obtained for $\eta_{\text {min }}$ $=5.68171$. The most stable static stellar configuration made of strange matter is given by quark stars with radius $R_{\text {stab }}$ $=9.97179 \times 10^{5} \mathrm{~cm}$ and with mass $M_{\mathrm{stab}}=0.96558 M_{\odot}$, corresponding to values of the central density of the order of $\rho_{C}=5.681 \times B=5.681 \times 10^{14} \mathrm{~g} / \mathrm{cm}^{3}$.

In the present paper we have obtained approximate formulas which give the dependence of the radius and mass of the static and rotating strange stars, built of self-bounded quark matter described by the MIT bag model, on the central 


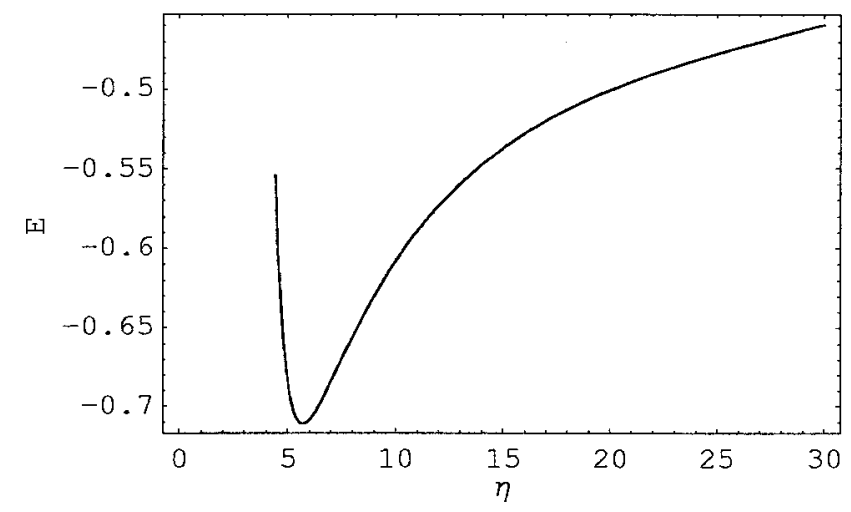

FIG. 6. Variation of the total energy $E$ (in the normalization $\left.c^{5} /(G)^{3 / 2} \sqrt{3 / 32 \pi B}=1\right)$ of the static strange quark star.

density of the stellar object and of its angular velocity. In the static case this is made possible due to the constancy of the chemical potential inside the star. The two unknown functions involved in the model must be obtained by fitting the exact formulas with data obtained from the numerical integration of the structure equations of the quark star. The resulting expression can reproduce the radius and mass of the static strange star with an error smaller than $1 \%$.

In the rotating case, with the use of the hydrostatic equilibrium condition that is the consequence of the Bianchi identities, we have also obtained mass-radius relations, depending on three functions describing the effect of rotation on strange star structure. These relations are exact in the sense that they have been obtained without any special assumptions. By assuming some appropriate forms for the unknown functions we have obtained a description of the mass and radius of the rotating strange stars which can reproduce the values obtained by numerical integration of the gravitational field equations with a mean error of around 3\%. The expressions of the unknown parameters have been chosen following a close analogy with the static case, whose relevance for the study of rotating general relativistic configurations seems to be more important than previously believed. These functions also incorporate some other general relativistic effects not explicitly taken into account, like the varia- tion of the moment of inertia of the star with the angular velocity. The authors of Ref. [41] have presented a formula valid for a broad range of realistic equations of state of dense matter expressing the moment of inertia in terms of stellar mass and radius. We have not used these results, obtained in the slow rotation limit because, at least in the case of strange stars, the Newtonian expression of the moment of inertia also leads to a quite accurate physical description of the rotating quark objects. As an application of the obtained formulas we had given a derivation of the "empirical" formula relating the maximum angular velocity to the mass and radius of the static quark star configuration. In the present paper we compared our results only with data obtained for maximally rotating (so-called "Keplerian models") so the given expressions could be not valid for stars rotating less rapidly. But a similar analysis leads to high-precision mass and radius formulas valid for a given angular velocity and central density range.

In the present analysis we restricted ourselves to the case of strange stars with superdense quark surface, built exclusively of strange quark matter (bare strange stars). In principle, one should also consider models of strange stars covered by an envelope (crust) consisting of nuclei immersed in an electron gas [3]. The nuclei, forming a crystal lattice of the crust, are separated from strange matter by a repulsive Coulomb barrier. The maximum mass of the crust was originally estimated to be $10^{-5} M_{\odot}[3]$, but more recent estimates give even a lower value $10^{-6} M_{\odot}[42]$. Within the version of slow rotation approximation the authors of Ref. [20] have shown that rapid rotation will increase the maximum mass of the strange star crust, but its effect on the mass and radius of the star is still expected to be small. Therefore, the formulas obtained in the present paper are also applicable for strange stars with crust with a very good approximation. Hence they give an accurate description of mass and radius of all stellartype quark-matter configurations.

\section{ACKNOWLEDGMENTS}

This work is partially supported by a RGC grant from the Hong Kong Government, K.S.C. was supported by a grant from the Croucher Foundation.
[1] E. Witten, Phys. Rev. D 30, 272 (1984).

[2] A. R. Bodmer, Phys. Rev. D 4, 1601 (1971); H. Tarazawa, INS report-338, INS, University of Tokyo, 1979.

[3] C. Alcock, E. Farhi, and A. Olinto, Astrophys. J. 310, 261 (1986).

[4] P. Haensel, J. L. Zdunik, and R. Schaeffer, Astron. Astrophys. 160, 121 (1986).

[5] For a recent review of basic properties of strange stars and astrophysical phenomena against or supporting the existence of strange stars, cf. K. S. Cheng, Z. G. Dai, and T. Lu, Int. J. Mod. Phys. D 7, 139 (1998).

[6] Z. G. Dai, Q. H. Peng, and T. Lu, Astrophys. J. 440, 815 (1995).

[7] K. S. Cheng and Z. G. Dai, Phys. Rev. Lett. 77, 1210 (1996).
[8] K. S. Cheng and Z. G. Dai, Phys. Rev. Lett. 80, 18 (1998).

[9] K. S. Cheng, Z. G. Dai, D. M. Wei, and T. Lu, Science 280, 407 (1998).

[10] Z. G. Dai and T. Lu, Phys. Rev. Lett. 81, 4301 (1998).

[11] X. D. Li, I. Bombaci, M. Dey, J. Dey, and E. P. J. van den Heuvel, Phys. Rev. Lett. 83, 3776 (1999).

[12] K. S. Cheng and Z. G. Dai, Astrophys. J. 492, 281 (1998).

[13] W. Zhang, A. P. Smale, T. E. Strohmayer, and J. H. Swank, Astrophys. J. Lett. 500, L171 (1998).

[14] W. Kluzniak, P. Michelson, and R. V. Wagoner, Astrophys. J. 358, 538 (1990).

[15] E. Farhi and R. L. Jaffe, Phys. Rev. D 30, 2379 (1984).

[16] P. Haensel and J. L. Zdunik, Nature (London) 340, 617 (1989).

[17] J. L. Friedman, J. R. Ipser, and L. Parker, Phys. Rev. Lett. 62, 
3015 (1989).

[18] E. Gourgoulhon, P. Haensel, R. Livine, E. Paluch, S. Bonazzola, and J.-A. Marck, Astron. Astrophys. 349, 851 (1999).

[19] M. Colpi and J. C. Miller, Astrophys. J. 388, 513 (1992).

[20] N. K. Glendenning and F. Weber, Phys. Rev. D 50, 3836 (1994).

[21] J. M. Lattimer, M. Prakash, D. Masak, and A. Yahil, Astrophys. J. 355, 241 (1990).

[22] N. Stergioulas, W. Kluzniak, and T. Bulik, Astron. Astrophys. (to be published).

[23] P. Haensel, M. Salgado, and S. Bonazzola, Astron. Astrophys. 296, 745 (1995).

[24] J.-P. Lasota, P. Haensel, and M. A. Abramowicz, Astrophys. J. 456, 300 (1996).

[25] L. D. Landau and E. M. Lifshitz, The Classical Theory of Fields (Pergamon, Oxford, 1975).

[26] L. D. Landau and E. M. Lifshitz, Statistical Physics (Pergamon, Oxford, 1980).

[27] T. K. Chu, M.Phil. thesis, University of Hong Kong, 1998.

[28] R. P. Kerr, Phys. Rev. Lett. 11, 237 (1963).

[29] R. Wijnands, M. van der Klis, M. Mendez, J. van Paradijs, W. H. G. Lewin, F. K. Lamb, B. Vaughan, and E. Kuulkers, Astrophys. J. Lett. 495, L39 (1998).
[30] P. G. Jonker, M. van der Klis, J. Homan, R. Wijnands, J. van Paradijs, M. Mendez, E. Kuulkers, and E. C. Ford, Astrophys. J. Lett. 511, L41 (1999).

[31] S. Bonazzola, E. Gourgoulhon, M. Salgado, and J.-A. Marck, Astron. Astrophys. 278, 421 (1993).

[32] R. Adler, M. Bazin, and M. Schiffer, Introduction to General Relativity (McGraw-Hill, New York, 1975).

[33] S. Chandrasekhar, The Mathematical Theory of Black Holes (Oxford University Press, Oxford, 1992).

[34] F. Weber and N. K. Glendenning, Phys. Lett. B 265, 1 (1991).

[35] F. Weber and N. K. Glendenning, Astrophys. J. 390, 541 (1992).

[36] J. B. Hartle and K. S. Thorne, Astrophys. J. 153, 807 (1968).

[37] B. Datta and R. C. Kapoor, Astrophys. J. 440, 815 (1988).

[38] N. Straumann, General Relativity and Relativistic Astrophysics (Springer, Berlin, 1984).

[39] D. Lynden-Bell and J. Katz, Mon. Not. R. Astron. Soc. 213, 21 (1985).

[40] O. Gron and S. Johannesen, Astrophys. Space Sci. 19, 411 (1992).

[41] D. G. Ravenhall and C. J. Pethick, Astrophys. J. 424, 846 (1994).

[42] Y. F. Huang and T. Lu, Astron. Astrophys. 325, 189 (1997). 